\title{
Fatores sociodemográficos: a interferência nos pacientes no período pós confecção de
}

\section{ostomias intestinais}

\author{
Sociodemographic factors: the interference in patients in the period after making intestinal \\ ostomies
}

Factores sociodemográficos: la interferencia en los pacientes en el período posterior a la realización de ostomías intestinales

\section{Resumo}

Objetivo: relacionar os principais fatores, internos e externos, que interferem no processo de readmissão hospitalar de pacientes ostomizados. Metodologia: estudo quantitativo e qualitativo, descritivo e de campo. Se aplicou, por meio online, um questionário a 23 participantes submetidos à confecção de ostomia intestinal em um Hospital Escola de Minas Gerais, Brasil. Perfizeram os critérios de inclusão pacientes submetidos à confecção de ostomia intestinal no período de 2015 a 2019. Foram excluídos pacientes que recusaram a participar da pesquisa e/ou que não possuíam contato telefônico. Os dados foram gerados no software Excel@ e realizado a análise descritiva e temática de conteúdo. Resultados: houve predominância no sexo feminino $14(60,9 \%)$, na faixa etária acima dos 50 anos 13 (56,5\%), com ensino fundamental completo $7(30,4 \%), 11(47,8 \%)$ com renda entre um e dois salários mínimos, grande parte $17(73,9 \%)$ possui acesso à Unidade de saúde. Também se notou, na maioria $(43,47 \%)$, dos pacientes o sentimento negativo quanto ao autocuidado com a ostomia. Conclusão - os principais fatores que interferem no processo de readmissão hospitalar são o baixo nível de escolaridade e a renda, tendo em vista que são fatores que dificultam o acesso à informação, fator fundamental para o cuidado adequado com a ostomia.

Palavras-chave: Colostomia; Estomia; Dados demográficos; Fatores sociais; Jejunostomia.

\begin{abstract}
Objective: to list the main factors, internal and external, that interfere in the hospital readmission process of ostomy patients. Methodology: quantitative and qualitative, descriptive and field study. A questionnaire was applied online to 23 participants who underwent an intestinal ostomy at a Teaching Hospital in Minas Gerais, Brazil. Patients who underwent intestinal ostomy in the period from 2015 to 2019 met the inclusion criteria. Patients who refused to participate in the research and/or who did not have telephone contact were excluded. Data were generated in Excel $\AA$ software and descriptive and thematic content analysis was performed. Results: there was a predominance of females $14(60.9 \%)$, aged over 50 years $13(56.5 \%)$, with complete primary education $7(30.4 \%), 11(47.8 \%)$ with income between one and two minimum wages, most $17(73.9 \%)$ have access to the Health Unit. It was also noticed, in the majority $(43.47 \%)$, of the patients the negative feeling about self-care with the ostomy. Conclusion - the main factors that interfere in the hospital readmission process are the low level of education and income, considering that these are factors that hinder access to information, a fundamental factor for proper care with the ostomy.
\end{abstract}

Keywords: Colostomy; Ostomy; Demographic data; Social factors; Jejunostomy.

\section{Resumen}

Objetivo: enumerar los principales factores, internos y externos, que interfieren en el proceso de reingreso hospitalario de pacientes ostomizados. Metodología: estudio cuantitativo y cualitativo, descriptivo y de campo. Se aplicó un cuestionario en línea a 23 participantes que se sometieron a una ostomía intestinal en un Hospital Universitario en Minas Gerais, Brasil. Los pacientes que fueron sometidos a ostomía intestinal en el período de 2015 a 2019 
cumplieron con los criterios de inclusión. Se excluyó a los pacientes que se negaron a participar en la investigación y / o que no tenían contacto telefónico. Los datos se generaron en el software Excel® y se realizó un análisis de contenido descriptivo y temático. Resultados: hubo predominio de mujeres 14 (60,9\%), mayores de 50 años 13 $(56,5 \%)$, con educación primaria completa $7(30,4 \%), 11(47,8 \%)$ con ingresos entre uno y dos salarios mínimos, la mayoría 17 (73,9\%) tiene acceso a la Unidad de Salud. También se notó, en la mayoría (43.47\%), de los pacientes el sentimiento negativo sobre el autocuidado con la ostomía. Conclusión - los principales factores que interfieren en el proceso de reingreso hospitalario son el bajo nivel de educación e ingresos, considerando que son factores que dificultan el acceso a la información, factor fundamental para el adecuado cuidado de la ostomía.

Palabras clave: Colostomía; Ostomía; Datos Demográficos; Factores sociales; Yeyunostomía.

\section{Introdução}

Este trabalho faz parte de um recorte do Mestrado Profissional em Saúde Ambiental e Saúde do Trabalhador Programa de Pós Graduação Saúde Ambiental e Saúde do Trabalhador (PPGAT), do Instituto de Geografia (IG) da Universidade Federal de Uberlândia (UFU).

As palavras ostomia, ostoma ou estomia derivam da palavra grega "estoma" e significam abertura de uma nova boca. Epidemiologicamente, existem no Brasil cerca de 400 mil ostomizados e anualmente surgem 10 mil novos casos (Ventura, 2020).

A ostomia intestinal de eliminação se refere à abertura cirúrgica no abdômen, na qual há a exteriorização de parte do segmento intestinal. A consistência das fezes varia com a porção do intestino na qual se realizou o procedimento cirúrgico (Ambe et al., 2018; Guideline, 2018). As principais causas para a realização deste procedimento são o câncer de intestino e doenças inflamatórias intestinais (Guideline, 2018). A confecção da ostomia é classificada como temporária ou permanente, de acordo com a causa e finalidade que a mesma foi construída. É realizada em porções diferentes do intestino, sendo as principais, colostomia e ileostomia (Oliveira et al., 2018).

As complicações deste tratamento podem ser classificadas em recentes ou tardias. Aquelas abrangem principalmente escoriação em pele, retração ou necrose do estoma, desidratação e escape do conteúdo colônico, que causam ferimentos à pele. Já as tardias englobam, principalmente, hérnia paraestomal, estenose, fístula, dermatite ou abcesso periostomal. Estas complicações geram hospitalizações e maiores taxas de readmissão, o que ocasiona elevados custos hospitalares (Messaris et al., 2012; Shabbir \& Britton, 2019).

A readmissão hospitalar consiste na admissão hospitalar de um paciente em um mesmo hospital, até 30 dias após sua alta. Tal conceito tem sido utilizado como indicador de desempenho nos sistemas de saúde, podendo refletir a qualidade da assistência, além de permitir o monitoramento de acesso inadequado ou excessivo às internações hospitalares (Dias, 2015).

Um estudo realizado na Flórida, nos EUA, evidenciou que de um total de 93.913 pacientes submetidos a algum tipo de ostomia, obteve-se uma taxa de readmissão, em até 30 dias, de 14,7\%. Os fatores relacionados à readmissão foram: raça não branca, idade inferior a 65 anos e pacientes com estomas. Instituições hospitalares com maior volume de atendimento apresentaram também as maiores taxas de readmissão, sendo o motivo mais comum a presença de infecção (Bliss et al., 2015).

Evidenciando as altas taxas de readmissões após confecção de ostomias intestinais, outra pesquisa, realizada em Madison nos Estados Unidos, por meio da análise de 43.903 prontuários de pacientes que realizaram colectomia ${ }^{1}$ entre 1992 e 2002 demonstrou que 4.662 (11\%) foram readmitidos (Greenblatt, 2010).

No Brasil, estudo realizado em 123 prontuários de pacientes que frequentaram o Ambulatório de Estomizados do Hospital Santa Casa de Misericórdia de Goiânia, no período de agosto de 2016 a agosto de 2017 mostrou que 9 (7\%) dos portadores de ostomias necessitaram de readmissão hospitalar (Oliveira et al., 2018).

\footnotetext{
${ }^{1}$ A colectomia consiste em um procedimento cirúrgico para retirada total ou de parte do cólon (Greenblatt, 2010).
} 
O estudo de Santos et al. (2019) realizado com 13 cônjuges de pessoas com estomias intestinais e cadastrados em um programa de dispensação de equipamentos coletores do interior de Minas Gerais, evidenciou que após a alta, pacientes estomizados apresentam dificuldades no desenvolvimento do autocuidado e isso resulta em readmissões hospitalares.

Sabe-se que a maioria das readmissões hospitalares são em decorrência de infecções pós operatórias. As mesmas se relacionam com os cuidados com o estoma, e sofrem influências de acordo com os fatores socioambientais como falta de saneamento básico, tipo de alimentação, baixa escolaridade e hábitos de vida (Batista et al., 2011; Greenblatt, 2010; Oliveira et al., 2018; Santos et al., 2019).

Sendo assim, a partir da vivência como Enfermeira Assistencial em uma Clínica Cirúrgica da instituição em estudo, e de acordo com os dados do setor de estatística do mesmo hospital, dos 197 pacientes que confeccionaram ostomia entre 2015 e 2019 pôde-se observar alto índice de pacientes com ostomias intestinais readmitidos no serviço por algum tipo de complicação, totalizando $46(23,35 \%)$. Estas complicações se mostraram das mais diversas formas, como prolapso ou desabamento do estoma, sepse, obstrução intestinal, especialmente em decorrência de fatores sociais como falta de higiene e cuidados domiciliares, falta de esclarecimentos a respeito da dieta adequada, entre outros.

Diante do exposto, apresentou-se como problema de estudo a seguinte questão: quais os fatores socioeconômicos e demográficos dos pacientes ostomizados que podem interferir nas readmissões hospitalares de pacientes com ostomias intestinais?

Com isso, este estudo objetiva apresentar o perfil sociodemográfico de pacientes ostomizados e relacionar os principais fatores que interferem no processo de readmissão hospitalar.

\section{Metodologia}

O estudo seguiu todos os preceitos éticos, sendo aprovado pelo Comitê de Ética da Universidade Federal de Uberlândia sob o $\mathrm{N}^{\circ}$ 4.175.805. Para resguardar a identidade dos participantes, estes foram codificados através de números de 1 a 23. Além disso, o Termo de Consentimento Livre e Esclarecido (TCLE) foi disponibilizado na plataforma do questionário. Para os participantes menores de 18 anos, o contato foi realizado com o responsável legal, que assinou o TCLE e respondeu o questionário com os dados do paciente submetido a ostomia.

A abordagem do estudo foi quantitativa e qualitativa, descritiva não experimental (Andrade, 2009; Creswell, 2007; Minayo, 2007). A pesquisa foi realizada em abril de 2021 com 23 pacientes do Hospital de Clínicas da Universidade Federal de Uberlândia (HC-UFU) que confeccionaram o ostoma entre 2015 - 2019 por meio da aplicação de um questionário, criado pela autora, com base em estudos já realizados (Ecco et al., 2018; Gomes \& Silva, 2013; Nascimento et al., 2018), com perguntas socioeconômicas, demográficas, de sentimentos e autocuidado com a ostomia.

Como critérios de inclusão adotou-se pacientes submetidos à confecção de ostomia intestinal no período de 2015 a 2019, e pacientes com os quais foi possível contato telefônico. Os critérios de exclusão, perfizeram-se de pacientes que recusaram participar da pesquisa e/ou que não possuíam número para contato ou prontuários incompletos.

De acordo com os dados de setor de estatística do HC-UFU (2020), o universo de pacientes que passou pela confecção de ostomia intestinal no período de 2015 a 2019 foi de 197 pacientes. Após busca ativa nos prontuários, no momento da pesquisa 114 pacientes haviam falecido (Uberlândia, 2020).

Devido à situação pandêmica, a qual orienta distanciamento social, foi necessário a mudança quanto a aplicação dos questionários. Assim, com relação aos de coleta de dados e instrumento de pesquisa, após contato prévio com os participantes, pelo aplicativo de mensagens WhatsApp ${ }^{\circledR}$, o questionário foi enviado por meio da plataforma Google Forms ${ }^{\circledR}$, sendo respondido online no período de 10 a 30 de abril de 2021. 
Os dados foram gerados no software Excel® e para as variáveis descritivas realizou-se a análise apresentada por meio de média e percentual e utilizou-se do software SPSS ${ }^{\circledR}$ versão 22.0. Já para os dados qualitativos, utilizou-se a Análise Temática de Conteúdo, proposta por Bardin (2016).

\section{Resultados e Discussão}

De acordo com os contatos por meio de mensagens no WhatsApp® e telefônico com 83 pacientes, dos quais 23 $(27,7 \%)$ responderam ao questionário, 9 (10,8\%) não se conseguiu contato, $7^{2}(8,4 \%)$ informaram não ter confeccionado ostomia e $44(51,8 \%)$ recusaram participar. A baixa adesão à pesquisa é evidenciada na Figura 1.

Figura 1: Fluxograma da justificativa das altas taxas de recusa

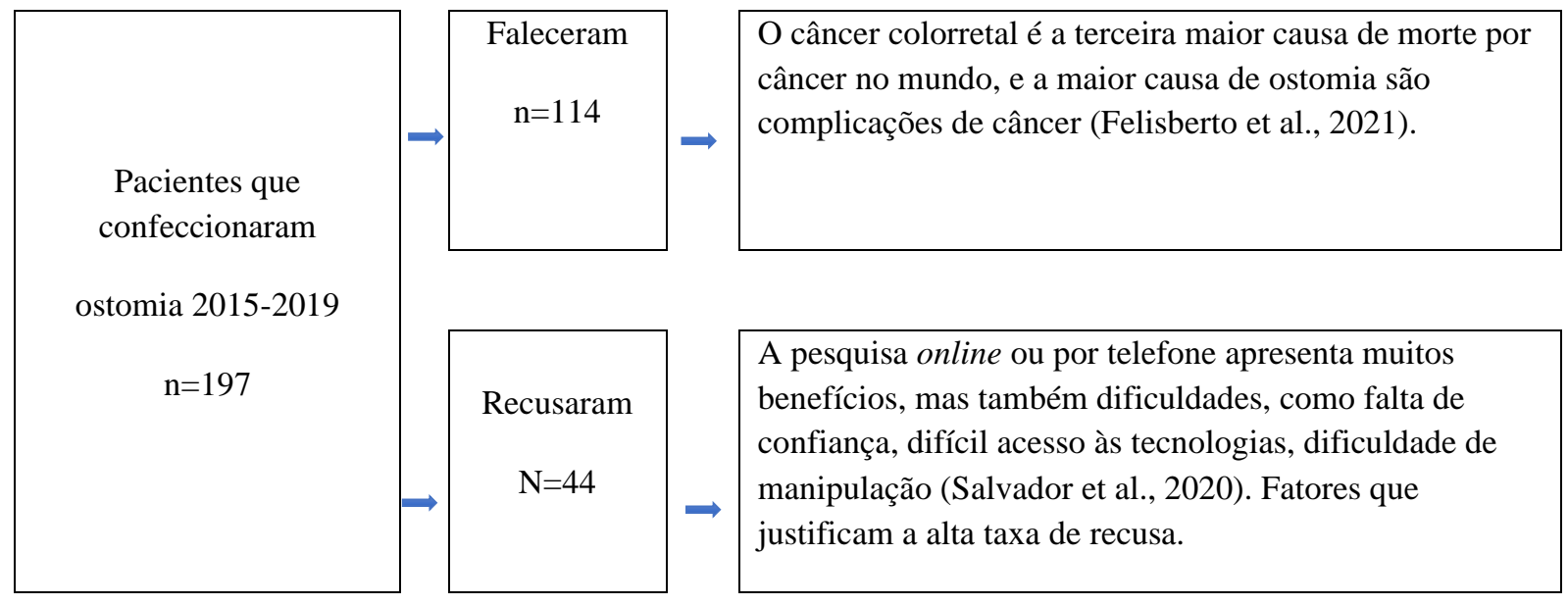

Fonte: Autores (2021).

O alto índice $(57,86 \%)$ de mortalidade dos pacientes ostomizados chamou atenção neste estudo. Tal fato pode ser em decorrência da causa para confecção da ostomia, cuja predominância, 34,8\%, foi câncer.

Observou-se, que entre os pacientes submetidos à confecção de ostomia intestinal no período de 2015 a 2019, há uma taxa equivalente a $57,9 \%$ de mortalidade e $51,8 \%$ de recusa na participação do estudo. A Figura 1 acima evidencia essa condição.

O câncer colorretal é a terceira maior causa de morte por de câncer no mundo, 45\% das pessoas diagnosticadas com essa patologia morrem (Felisberto et al., 2021). De acordo com dados epidemiológicos do Instituto Nacional do Câncer (INCA, 2020), estima-se que em 2020 surgiram 41.010 casos novos de câncer de intestino. Além disso, um dos fatores de risco que permite o desenvolvimento deste tipo de câncer é possuir 50 anos ou mais, por isso a idade pode influenciar na confecção da ostomia.

Em relação ao aumento da taxa de mortalidade, o estudo de Greenblatt (2010) mostrou que a readmissão hospitalar está fortemente associada a esse aumento. A taxa de mortalidade para pacientes readmitidos foi de 26,6\%, e de 11,0\% para pacientes não readmitidos.

A mortalidade dos pacientes ostomizados, às vezes, se apresenta após complicações com a ostomia. Porém, fatores ambientais como local de moradia não se relacionam tanto com estas elevadas taxas, a não ser quando relacionados à falta de saneamento básico (Greenblatt, 2010). Além disso, a maioria dos participantes $17(73,9 \%)$ tem acesso fácil à UBS, assim os fatores ambientais têm pouco impacto nos cuidados com a ostomia.

\footnotetext{
${ }^{2} \mathrm{O}$ setor de estatística forneceu os dados dos pacientes que constavam como ostomizados no sistema do Hospital, mas estes pacientes que não realizaram ostomias não tiveram seus prontuários atualizados, por isso foram contatados. E, seguindo os critérios de exclusão, não participaram do estudo.
} 
Em um paradoxo, fatores sociodemográficos e orgânicos como baixa escolaridade, falta de conhecimento, falta de apoio e prognóstico ruim da doença dificultam os cuidados com o estoma, assim como nos hábitos de vida. E, consequentemente impactam de maneira significativa nas altas taxas de readmissão hospitalar e morte (Greenblatt, 2010; Bliss et al., 2015; Ecco et al., 2018).

Uma das caracterizações realizadas com os 23 entrevistados(as) ocorreu a partir da coleta de informações, relacionadas às características demográficas, seus percentuais relacionados ao sexo, idade e estado civil como demonstra a Tabela 1.

Tabela 1: Características sociodemográficas.

\begin{tabular}{lcc}
\hline Característica & n (23) & Percentual \% \\
\hline Sexo & 14 & 60,9 \\
Feminino & 9 & 39,1 \\
Masculino & & 13,0 \\
Faixa etária & 3 & 30,4 \\
$0-5$ anos & 7 & 56,5 \\
$20-50$ anos & 13 & \\
Acima de 50 anos & & 34,8 \\
Estado Civil & 8 & 47,8 \\
Solteiro (a) & 11 & 13,0 \\
Casado (a) & 3 & 4,4 \\
Divorciado (a) & 1 & \\
Viúvo (a) & & \\
\hline
\end{tabular}

Fonte: Autores (2021).

Em relação aos dados sociodemográficos, apresentados na Tabela 1, nota-se predominância do sexo feminino, a faixa etária acima de 50 anos e casados/as.

A análise da variável sexo ocorreu em estudos (Diniz et al., 2020; Oliveira et al., 2018; Valau et al., 2020) realizados nas regiões Nordeste, Sudeste e Sul respectivamente e mostraram predomínio do sexo masculino. Tal circunstância pode relacionar-se ao fato de o homem procurar menos os serviços de saúde para a prevenção de agravos. O presente estudo se opõe a literatura consultada, na qual há predominância do sexo feminino, 14 (60,9\%), sugerindo estar relacionada ao número de pessoas ostomizadas que recusaram participar do estudo $(n=43)$ das quais $31(72,0 \%)$ eram do sexo masculino, evidenciando assim essa redução da participação masculina. Associado a isso, encontram-se nos homens a dificuldade em verbalizar as necessidades, já que falar das suas adversidades de saúde pode indicar uma provável demonstração de fraqueza e feminilização diante das outras pessoas (Silva et al., 2013).

A média da faixa etária foi 46 anos, sendo a mínima 2 e a máxima 84, a maior frequência observada 13 (56,5\%) foi na categoria acima dos 50 anos. Esses achados estão em consonância com estudos (Diniz et al., 2020; Melo et al., 2018; Miranda et al., 2016) os quais apresentam a faixa etária acima de 50 anos como a mais acometida para a confecção de ostomias. Tal fato pode estar relacionado ao avançar da idade, que consiste em uma das condições que favorece a oncogênese, em decorrência dos maus hábitos de vida, provocando o aumento da taxa de confecção de ostomias na população mais velha.

A maioria, $11(47,8 \%)$ dos participantes eram casados e quem realizava os cuidados com a ostomia era um familiar 15 $(65,2 \%)$. Assim, ter alguém pertencente ao seu núcleo familiar pode demonstrar suporte à pessoa ostomizada, já que a presença 
do (a) companheiro (a) e/ou outro familiar, perante essa condição crônica, pode auxiliar na adaptação e enfrentamento da nova situação de vida (Silva et al., 2017).

A Tabela 2 apresenta a relação entre a escolaridade, a categoria profissional e o tipo de vínculo empregatício.

Tabela 2: Relação entre a escolaridade, categoria profissional e vínculo empregatício.

\begin{tabular}{|c|c|c|c|c|}
\hline Escolaridade & $\mathbf{N}(\mathbf{2 3})$ & $\%$ & Categoria Profissional & Vínculo \\
\hline \multirow[t]{6}{*}{ Fundamental Incompleto } & 1 & 4,4 & Aposentado & Outros \\
\hline & 2 & 8,8 & Do Lar & \\
\hline & 3 & 13.0 & Sem Profissão & \\
\hline & 1 & 4,4 & Cozinheiro & \\
\hline & 1 & 4,4 & Granieiro & CLT \\
\hline & 1 & 44 & Auxiliar de Produção & \\
\hline Fundamental Completo & 1 & 4,4 & Produtor Rural & Autônomo \\
\hline \multirow[t]{2}{*}{ Médio Incompleto } & 1 & 4,4 & Do Lar & Outros \\
\hline & 1 & 4,4 & Costureira & CLT \\
\hline \multirow[t]{6}{*}{ Médio Completo } & 1 & 4,4 & Corretor de Imóveis & Autônomo \\
\hline & 1 & 4,4 & Costureira & \\
\hline & 1 & 4,4 & Despachante & \\
\hline & 1 & 4,4 & Escritora & \\
\hline & 1 & 4,4 & Vendedora & CLT \\
\hline & 1 & 4,4 & Aposentado & Outros \\
\hline \multirow[t]{2}{*}{ Superior Incompleto } & 1 & 4,4 & Empreendedor & Autônomo \\
\hline & 1 & 4,4 & Técnico em Química & \\
\hline Superior Completo & 1 & 4,4 & Advogada & Autônomo \\
\hline \multirow[t]{2}{*}{ Pós graduação } & 1 & 4,4 & Estudante & Outros \\
\hline & 1 & 4,4 & Educador Físico & Autônomo \\
\hline
\end{tabular}

Fonte: Autores (2021).

Vale a pena ressaltar que na Tabela 2, relacionado ao nível de escolaridade, percebeu-se uma predominância de pessoas com ensino fundamental incompleto, sendo que os vínculos empregatícios como estudantes, donas de casas, desempregados e trabalhadores informais classificados como outros também apresentaram predominância.

O nível de escolaridade pode ser um importante fator para a não prevenção e cuidado, tendo em vista que a escolaridade influencia positivamente na aprendizagem sobre os cuidados adequados com a ostomia - os quais são essenciais para o manejo do autocuidado com consequente adaptação e prevenção de complicações (Melo et al., 2018; Miranda et al., 2016).

A soma da renda, do número de moradores e da condição da casa são evidenciadas no Gráfico 1. 
Gráfico 1: Relação entre participantes, número de moradores, soma da renda dos moradores e tipo de casa.

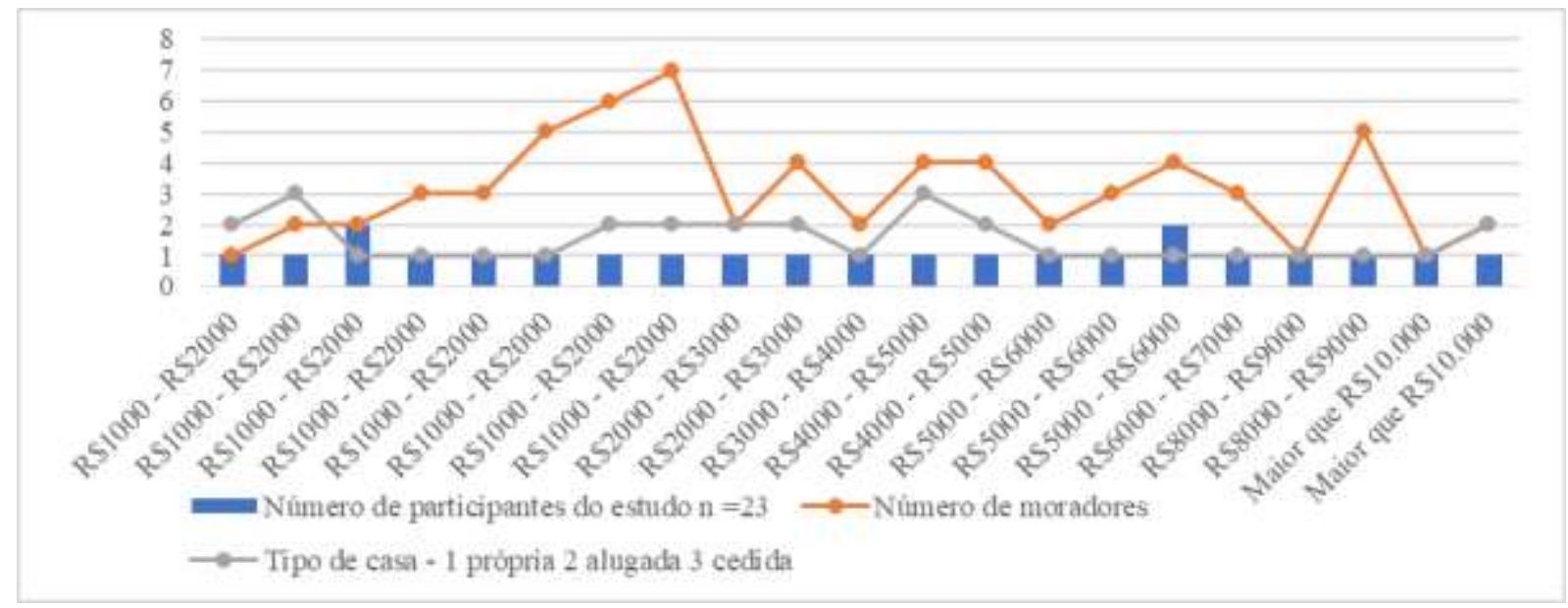

Fonte: Autores (2021).

No Gráfico 1 deve-se observar que no que cerne às características domiciliares 14 (60,9\%) possuem casa própria, 7 $(34,8 \%)$ vivem de aluguel e $2(8,7 \% \%)$ tem a moradia cedida. Em relação ao acesso à água e esgoto tratados 1 (4,4\%) informou não possuir. Em relação à renda individual, observou-se que 5 (21,8\%) recebem menos de $\mathrm{R} \$ 1000,11(47,8 \%)$ possuem renda entre $\mathrm{R} \$ 1000$ e $\mathrm{R} \$ 2000,1$ (4,4\%) entre $\mathrm{R} \$ 3000$ e $\mathrm{R} \$ 4000,4(17,4 \%)$ entre $\mathrm{R} \$ 4000$ e 5000, $2(8,7 \%)$ maior que $\mathrm{R} \$ 5000$ e 1 (4,4\%) maior que $\mathrm{R} \$ 10000$ (Gráfico 1). O nível de escolaridade predominante 7 (30,4\%) foi o fundamental completo e a renda de $11(47,8 \%)$ pacientes entre um e dois salários mínimos. Estabelecendo a relação entre renda e escolaridade e coadunando com os estudos de Diniz et al. (2020) e Valau et al. (2020) predominaram a baixa escolaridade e a renda entre um e dois salários mínimos nas pessoas com ostomia intestinal.

Nos cuidados com a ostomia, a baixa renda pode influenciar na dificuldade de aquisição de materiais adequados e de qualidade. Muitas vezes, estes não são fornecidos pelas instituições em que o ostomizado se encontra cadastrado, gerando assim um problema no tratamento e cuidado para os aqueles que não têm condições de comprar (Menezes et al., 2013).

O Gráfico 2 apresenta os hábitos de vida dos participantes do estudo.

Gráfico 2 - Informações acerca do número de refeições diárias realizadas pelos participantes n (23).

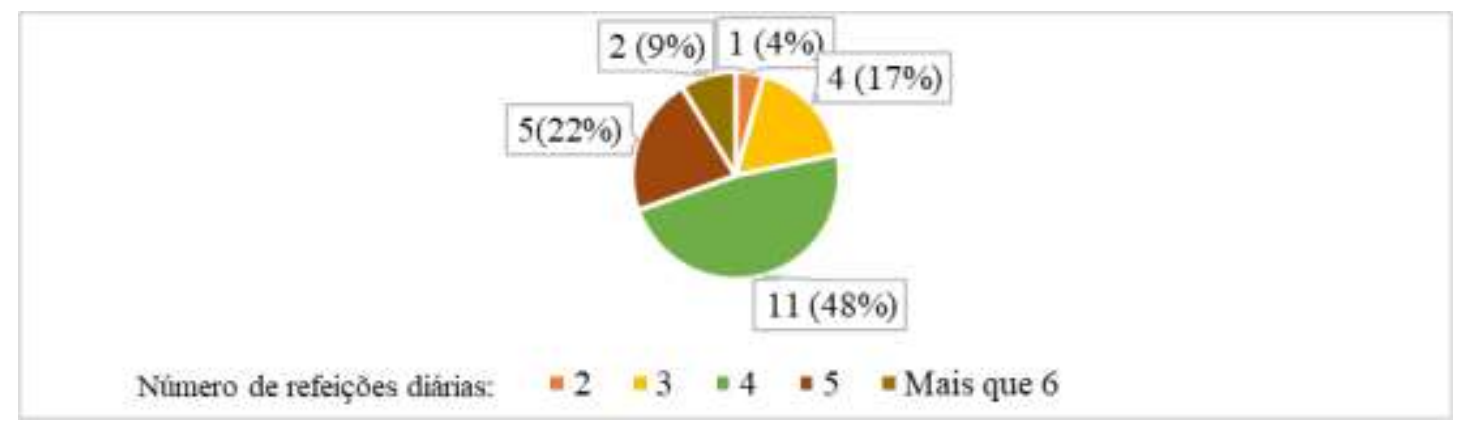

Fonte: Autores (2021).

Em relação aos estilos de vida dos entrevistados (as), foram divididos em dois contextos. No primeiro quesito 21 $(92,3 \%)$ relataram não serem tabagistas e não ingerirem bebida alcoólica 20 (86,9\%). O outro, tratou do acesso à alimentação, em que $20(86,9 \%)$ relataram apresentar alimentação adequada, embora em relação ao número de refeições por dia tenha sido muito variável (Gráfico 2). 
Sabe-se que a confecção de uma estomia ocasiona uma grande mudança na vida dos pacientes. As alterações biológicas somam-se à nova rotina de cuidados, que deve aderir hábitos saudáveis de alimentação, de higiene e de condições de vida (Salomé et al., 2015, Moreira et al., 2017). Assim, neste estudo, muitos participantes 5 (21,7\%) embora possuam baixa renda $^{3}$ e escolaridade $49(39,13)$, apresentam hábitos saudáveis o que proporciona melhora da condição.

Quanto aos cuidados com a ostomia, foram realizados pelo próprio paciente por $6(26 \%)$ deles, $15(65,2 \%)$ os familiares que realizavam esta função e em $2(8,69 \%)$ o manejo foi realizado por cuidador, $13(56,5 \%)$ se sentiam aptos a cuidar da ostomia. Assim, ter alguém pertencente ao seu núcleo familiar pode demonstrar auxílio à pessoa ostomizada, já que a segurança em ter uma pessoa próxima, perante essa condição crônica, pode assessorar nos processos da nova situação de vida (Silva et al., 2017).

Quando se trata do procedimento de confecção de ostomia a maioria 7 (34,8\%) necessitou dela em decorrência de câncer. A predominância do tipo de ostomia foi colostomia em 14 (60,9\%) e a ileostomia em 9 (39,1\%).

O Gráfico 3 apresenta as principais causas de readmissão após confecção de ostomia intestinal.

Gráfico 3 - Causas da readmissão dos pacientes ostomizados.

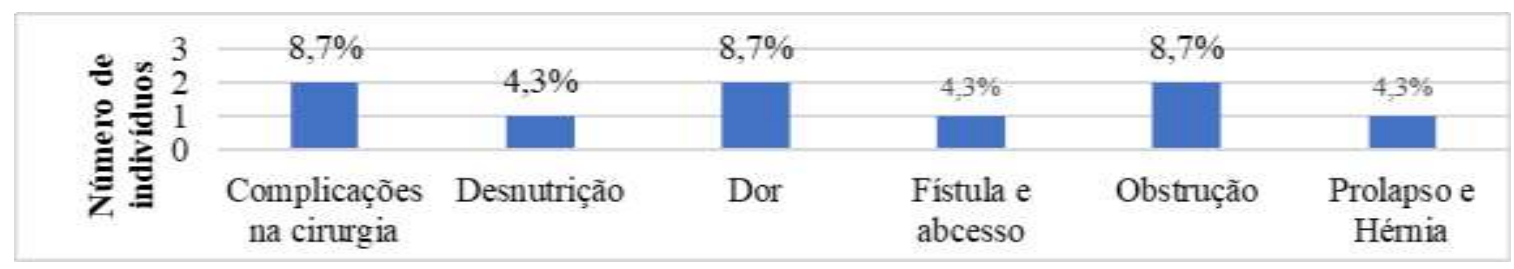

Fonte: Autores (2021).

Embora, grande parte dos ostomizados $8(34,8 \%$,) tenham relatado conhecimento e preparo para realizar os cuidados, a taxa de readmissão foi alta, 39,1\%, sendo causada por diversos fatores, e, dos pacientes readmitidos $34,8 \%$ necessitaram de nova intervenção cirúrgica. O conhecimento e cuidado são essenciais para evitarem-se as readmissões, tendo em vista que diminui os riscos de complicações (Gráfico 3).

O baixo nível de escolaridade pode impactar no déficit de conhecimento acerca das medidas preventivas para as complicações das ostomias de eliminação intestinal (EIE) e aumentar as taxas de readmissões (Nascimento et al., 2018). Além disto, as readmissões hospitalares dos pacientes com EIE estão associadas ao aumento das taxas de mortalidade. Suas causas são potencialmente evitáveis por meio de instruções adequadas e acessíveis, relacionadas ao processo de cuidado (Greenblatt et al., 2010). Em virtude disto, é fundamental, tanto em âmbito hospitalar quanto exterior a ele, o desenvolvimento de ações em saúde, implementadas pela equipe multiprofissional, que abordem de maneira clara e acessível os cuidados e manejo com as ostomias de eliminação intestinal, a fim de se evitar as principais causas de readmissão (Nascimento et al., 2018; Greenblatt et al., 2010).

Para o aspecto qualitativo da pesquisa, observaram-se algumas emoções quanto ao autocuidado, suscitadas após a confecção da ostomia, e que foram apresentadas durante a aplicação do instrumento. Para as crianças que participaram do estudo 3 (100\%) foram respondidos pela mãe, que alegaram o fato de as crianças, à época da ostomia, serem bebês e não entenderem a dinâmica situacional, não demonstravam sentimentos. Em relação aos adultos houve predominância 10 (43,47\%) de sentimentos negativos relacionados à confecção da ostomia, conforme as respostas a seguir: "É muito incômodo e constrangimento não conseguimos ter o autocuidado." (Paciente 15). "Sentimento de insatisfação com próprio corpo de limitação, constrangimento, impotência em relação à vida normal após colostomia." (Paciente 21). "No primeiro momento

\footnotetext{
${ }^{3}$ Famílias de baixa renda são as que apresentam renda per capta até R\$522,00 (BRASIL, 2018).
}

${ }^{4}$ Baixa escolaridade são pessoas que não concluíram o ensino fundamental (IBGE, 2017). 
tristeza com a nova condição." (Paciente 4). "Assim que saí do hospital, era de nojo, parecia que estava fedendo." (Paciente 8). "Medo de machucar ou de manusear errado." (Paciente 22). "Sofrimento e Alívio." (Paciente 29). "No início tristeza." (Paciente 16). "Sem aceitação." (Paciente 12). "No começo deu uma pequena depressão, mas depois fui me adaptando." (Paciente 6). "Difícil.” (Paciente 20)

Notou-se também a presença de relatos de falta de conhecimento, apresentados nas narrativas. "Não sabia como funcionava". (Paciente 3). "Não consigo". (Paciente 7).

Corroborando com a pesquisa, estudo realizado com 30 ostomizados da Associação Cearense de Ostomizados, evidenciou dificuldades de autocuidado e sentimentos negativos com a ostomia (Menezes et al., 2013).

Pesquisa realizada em Uberaba-MG, com 23 pessoas ostomizadas há mais de dois anos e cadastradas no Programa de Assistência Multidisciplinar ao Paciente Ostomizado mostrou que grande número de pacientes não se adaptou à ostomia e adotou atitudes de negação, gerando sentimentos depreciativos assim como problemas emocionais, sociais e psicológicos (Coelho et al., 2013).

Diante do exposto nesta pesquisa, a presença de tantos sentimentos negativos e insegurança são preocupantes, tendo em vista que, muitas vezes, estes sentimentos são provocados pelas mudanças ocasionadas pelo procedimento, mas também pela falta de conhecimento. Situações que acabam gerando desequilíbrios de ordem psicológica, emocional e social, ocasionando déficits nos cuidados com a ostomia e consequentes complicações, que podem levar a readmissões hospitalares e elevação nas taxas de mortalidade (Coelho et al., 2013; Menezes et al., 2013; Salomé et al., 2017)

Constituíram-se limitações para este estudo a não aceitação do público masculino em participar do estudo, pois muitas vezes esse gênero tem dificuldade em verbalizar seus anseios, prejudicando assim maiores interpretações. Além disso, surgiram também dificuldades relacionadas com a situação pandêmica, que devido à orientação de distanciamento social ocasionou a mudança quanto à aplicação dos questionários - estes seriam aplicados de forma presencial, oferecendo, principalmente, aos pacientes com baixa escolaridade, maior segurança e chance de esclarecimentos. Acredita-se que em decorrência dos golpes cibernéticos divulgados na mídia, associados à falta de conhecimento e medo do crime digital pelos participantes, a aplicação dos questionários de maneira online tenha provocado o alto índice de recusa.

\section{Conclusão}

O perfil sociodemográfico de pacientes ostomizados apresentou alta taxa de mortalidade, sendo a maioria do sexo feminino, casados, ensino fundamental completo, renda entre um e dois salários mínimos e trabalhadores autônomos.

De acordo com os resultados encontrados na pesquisa e a relação com a literatura, os principais fatores que interferem no processo de readmissão hospitalar são o baixo nível de escolaridade e a renda, tendo em vista que, são fatores que dificultam o acesso à informação - fator fundamental para o cuidado adequado com a ostomia.

Outra circunstância importante, que pode influenciar no processo de readmissão, é a presença de sentimentos negativos provocados pelas mudanças de hábitos e auto-imagem ocasionadas pela ostomia, que acabam gerando desequilíbrios psicológicos, emocionais e sociais, e que podem dificultar o processo de autocuidado.

A relevância desse estudo é que os resultados podem subsidiar a criação de um protocolo institucional de atendimento e acompanhamento ao paciente ostomizado, otimizando a assistência aos internados no Hospital de Clínicas de Uberlândia. Para isso, apontamos como propostas o desenvolvimento de intervenções constantes, de educação continuada e permanente bem como instruções de técnicas de cuidado e manejo, assim como orientações quanto aos hábitos de vida, além do acompanhamento psicológico, que promovam nestes pacientes a adesão adequada aos cuidados com a ostomia. 
Por isso, é importante a implantação de uma equipe multidisciplinar, mas integrada na unidade estudada, com foco nos pacientes ostomizados e que informe e divulgue toda a rede de apoio existente e destinada aos cuidados e orientações aos pacientes ostomizados.

Para trabalhos futuros sugerimos implantação e avaliação de protocolos e educação contínua e permanente. E para esse processo é fundamental a inclusão dos pacientes com ostomias de eliminação intestinal, para apontarem suas dificuldades, anseios e o que pode ser feito para melhorar as condições.

\section{Referências}

Ambe, P. C., Kurz, N. R., Nitschke, C., Odeh, S. F., Möslein, G., \& Zirngibl, H. (2018). Intestinal Ostomy: classification, indications, ostomy care and complication management. Dtsch Arztebl, 115(11), 182-187. https://doi.org/10.3238/arztebl.2018.0182.

Andrade, M. M. (2009). Introdução a metodologia do trabalho científico. Atlas.

Bardin, L. (2016). Análise de conteúdo. Edições.

Batista, F. M. L. R., Rocha, F. C. V., Silva, D. M. G., \& Silva, F. J. G. (2011) Autoimagem de clientes com colostomia em relação à bolsa coletora. Revista Brasileira de Enfermagem, 64(6), p.1043-1047. https://doi.org/10.1590/S0034-71672011000600009

Bliss, L. A. M. D., Maguire, L. H. M. D.; Chau, Z. M. D., Yang, C. J. M. B. C. B., Nagle, D. A. M. D., Chan, A. T. M. D., \& Tseng, J. F. M. D. (2015). Readmission After Resections of the Colon and Rectum: Predictors of a Costly and Common Outcome. Diseases of the Colon \& Rectum, 58(12), p.1164-1173. https://doi.org/10.1097/DCR.0000000000000433

Brasil. (2018). O que é cadastro único? Secretaria de desenvolvimento Social. https://www.sedes.df.gov.br/cadastro-unico/

Creswell, J. W. (2007). Projeto de pesquisa: métodos qualitativo, quantitativo e misto. Artmed.

Coelho, A. R., Santos, F. S., \& Poggetto, M. S. D. (2013). A estomia mudando a vida: enfrentar para viver. Revista Mineira de Enfermagem, 17(2). http://www.dx.doi.org/10.5935/1415-2762.20130021

Ecco, L., Dantas, F. G., Melo, M. D. M., Freitas, L. S., Medeiros, L. P., \& Costa, I. K. F. (2018). Perfil de pacientes colostomizados na Associação dos Ostomizados do Rio Grande do Norte. ESTIMA, Brazilian Jornaul Enterostomal Therapy., 16(e0518). https://doi.org/10.30886/estima.v16.351_PT

Dias, B. M. (2015). Readmissão hospitalar como indicador de qualidade [Dissertação mestrado, Escola de Enfermagem, Universidade de São Paulo]. https://doi.org/10.11606/D.22.2016.tde-22122015-101155.

Diniz, I. V., Barra, I. P., Silva, M. A., Oliveira, S. H. S., Mendonça, A. E. O., \& Soares, M. J. G.O. (2020). Perfil epidemiológico de pessoas com estomias intestinais de um centro de referência. ESTIMA, Brazilian Jornaul Enterostomal Therapy, 18(1), p.1-7. https://doi.org/10.30886/estima.v18.929_IN.

Felisberto, Y. S., Santos, C. D. P. C., Caires, P. T. P. R. C., Bitencourt, A. C. O., Mendes, A. V. F. D., Pinho, J. M. B. L., Oliveira, R. A. L., Castro, B. T., Oliveira, P. M. R., \& Santos, J. M. (2021). Câncer colorretal: a importância de um rastreio precoce. Revista Eletrônica Acervo Saúde, 13(4), p.1-7. https://doi.org/10.25248/reas.e7130.2021.

Guideline Development Task Force, \& Wound, Ostomy and Continence Nurses Society (2018). WOCN Society Clinical Guideline: Management of the Adult Patient with a Fecal or Urinary Ostomy-An Executive Summary. Journal of Wound, Ostomy and Continence Nursing, 45(1), 50-58. https://doi.org/10.1097/WON.0000000000000396

Gomes, N. S., \& Silva, S. R. (2013). Avaliação da autoestima de mulheres submetidas à cirurgia oncológica mamária. Texto e Contexto Enfermagem, 22(2), p.509-519, 2013.

Greenblatt, D. Y., Weber, S. M., O'Connor, E. S., LoConte, N. K., Liou, J. I., \& Smith, M. A. (2010). Readmission after colectomy for cancer predicts oneyear mortality. Ann Surgery, 251(4), 659-669. https://doi.org/10.1097/SLA.0b013e3181d3d27c

Instituto Brasileiro de Geografia. (2017). Pesquisa nacional por amostra de domicílios contínua. Instituto Brasileiro de Geografia IBGE. https://www.ibge.gov.br/estatisticas/sociais/trabalho/17270-pnad-continua.html?=\&t=o-que-e

Instituto Nacional do Câncer José Alencar Gomes da Silva. (2020). Câncer de Intestino. Instituto Nacional do Câncer José Alencar Gomes da Silva INCA. https://www.inca.gov.br/numeros-de-cancer

Melo, M. D. M., Silva, I. P., Oliveira, D . M. S., Medeiros, A. S. A., Souza, A. J. G., \& Costa, I. K. F. (2018). Association of sociodemographic and clinical characteristics with the self-esteem of stomized persons. Revista Mineira em Enfermagem, 22(e-1076). https://doi.org/10.5935/1415-2762.20180006.

Menezes, L. C. G., Guedes, M. V. C., Oliveira, R. M., Oliveira, S. K. P., Meneses, L. S. T., \& Castro, M. E. (2013). Prática de autocuidado de estomizados: contribuições da teoria de Orem. Revista da Rede de Enfermagem do Nordeste, 14(2), 301-310.

Messaris, E., Sehgal, R. M. B., Deiling, S. B. A.; Koltun, W. A. M. D., Stewart, D., McKenna, K., \& Poritz, L. S. (2012). Dehydration is the most common indication for readmission after diverting ileostomy creation. Diseases of the Colon Rectum, 55(2), 175-180. https://doi.org/10.1097/DCR.0b013e31823d0ec5.

Minayo, M. C. S. (2007). O desafio do conhecimento. Hucitec. 
Research, Society and Development, v. 11, n. 1, e43711125227, 2022

(CC BY 4.0) | ISSN 2525-3409 | DOI: http://dx.doi.org/10.33448/rsd-v11i1.25227

Miranda, S. M., Luz, M. H. B. A., Sonobe, H. M., Andrade, E. M. L. R., \& Moura, E. C. C. (2016). Caracterização Sociodemográfica e Clínica de Pessoas com Estomia em Teresina. ESTIMA, Brazilian Jornaul Enterostomal Therapy, 14(1). https://doi.org/10.5327/Z1806-3144201600010005

Moreira, L. R., Souza, J. C., Oliveira, M. M., Melo, N. S., \& Cerqueira, T. F. (2017). Autocuidado com estomias: compreensão de pacientes hospitalizados acerca das orientações recebidas pela equipe. Enfermagem Revista, 20(2).

NASCIMENTO, M. V. F., Vera, O. S., Silva, M. C. R., Morais, F. F., Andrade, E. M. L. R., \& Bastos, S. N.. M. A. N. (2018). Perfil sociodemográfico e clínico de pacientes em pós operatório de confecção de estomias intestinais de eliminação. Ciencia y enfermería, 24(15).

Oliveira, I. V., Silva, M. C., Silva, E. L., Freitas, V. F., Rodrigues, F. R., \& Caldeira, L. M. (2018). Cuidado e saúde em pacientes estomizados. Revista Brasileira em Promoção da Saúde, 31(2), 1-9. https://doi.org/10.5020/18061230.2018.7223.

Salomé, G. M., Lima, J. A., Muniz, K. C., Faria, E. C., \& Ferreira, L. M. (2017). Lócus de controle em saúde, imagem corporal e autoestima nos indivíduos com estoma intestinal. Journal of Coloproctology, 35(3), 168-174. https://doi.org/10.1016/j.jcol.2017.04.003

Salomé, G. M., Almeida, S. A., Mendes, B., Carvalho, M. R. F., \& Massahud, M. R. (2015). Assessment of subjective well-being and quality of life in patients with intestinal stoma. Journal of Coloproctology, 37(3). https://doi.org/10.1016/j.jcol.2015.03.002

Santos, F. S., Vicente, N. G.,Bracarense, C. F., Dal-Poggeto, M. T., Goulart, B. F., \& Rodrigues, L. R. (2019). Percepção dos cônjuges de pessoas com estomia intestinal sobre a sexualidade do casal. Revista Mineira de Enfermagem, 23(e-1217). https://doi.org/10.5935/1415-2762.20190065

Shabbir, J., \& Britton, D. C. (2010). Stoma complications: a literature overview. Colorectal Disease, 12(10), 958-964. https://doi.org/10.1111/j.14631318.2009.02006.x

Silva, C. R. D. T., Andrade, E. L. M. R., Luz, M. H. B. A., Andrade, J. X., \& Silva, G. R. F. (2017). Qualidade de vida de pessoas com estomias intestinais de eliminação. Revista Acta Paulista de Enfermagem, 30(2), 144-151. https://doi.org/10.1590/1982-0194201700023

Silva, P. L. N., Moreira, M. M. Saiberth, C. C., Santos, s., \& Souza, J. R. (2013). Política de atención a lasaluddelhombreen Brasil y los retos de suimplantación: una revisión integrativa. Enfermèria globlal, 12(32), 81-413.

Uberlândia. (2020). Pacientes de Uberlândia submetidos à confecção de ostomias entre 2015 e 2019. Gestão de Informações Hospitalares, Setor de Estatísticas e informações hospitalares.

Valau, C. A. D., Simon, B. S., Garcia, R. P., Dalmolin, A., Stamm, B, \& Harter, J. (2020). Perfil sociodemográfico e práticas de autocuidado desenvolvidas por pessoas com estomia intestinal de eliminação. The Brazilian Journal of Development, 6(6), 41030-41047, 2020. https://doi.org/10.34117/bjdv6n6-588

Ventura, L. A. S. (2020). Portaria do SUS para pessoas ostomizadas precisa de atualização. https://brasil.estadao.com.br/blogs/vencer-limites/portaria-dosus-para-pessoas-ostomizadas-precisa-de-atualizacao/ 